\title{
Polyakov line actions from SU(3) lattice gauge theory with dynamical fermions: first results via relative weights
}

\author{
Roman Höllwieser ${ }^{* 1,2}$ and Jeff Greensite ${ }^{3}$ \\ E-mail: hromanakph.tuwien.ac.at, greensitasfsu.edu \\ ${ }^{1}$ Department of Physics, New Mexico State University, PO Box 30001, Las Cruces, NM \\ 88003-8001, USA \\ ${ }^{2}$ Institute of Atomic and Subatomic Physics, Vienna University of Technology, Operngasse 9, \\ 1040 Vienna, Austria \\ ${ }^{3}$ Physics and Astronomy Department, San Francisco State University, San Francisco, CA 94132, \\ USA
}

\begin{abstract}
We apply the relative weights method to extract an effective Polyakov line action, at finite chemical potential, from an underlying SU(3) lattice gauge theory with dynamical fermions. The center-symmetry breaking terms in the effective theory are fit to a form suggested by the hoppingparameter expansion, and the effective action is solved at finite chemical potential by a mean field approach. We present preliminary results for one-link staggered fermions with mass $m a=1.0$ and Wilson gauge action at $\beta=5.4$ on $L^{3} \times 4$ lattices with $L=16$. $^{\dagger}$
\end{abstract}

The 33rd International Symposium on Lattice Field Theory

14 - 18 July 2015

Kobe International Conference Center, Kobe, Japan

*Speaker.

${ }^{\dagger}$ The numerical simulations were performed at the Phoenix and Vienna Scientific Cluster (VSC) at VUT and the Riddler Cluster at NMSU. This research was supported by the Erwin Schrödinger Fellowship program of the Austrian Science Fund FWF ("Fonds zur Förderung der wissenschaftlichen Forschung") under Contract No. J3425-N27 (R.H.) and the U.S. Department of Energy under Grant No. DE-FG03-92ER40711 (J.G.). 


\section{Introduction}

We present our approach to the sign problem in $\mathrm{QCD}$, see e.g. [1, 2], which is to map QCD with a chemical potential into a simpler effective theory, namely, the effective Polyakov line action (PLA). The effective Polyakov line action $S_{P}$ can be computed analytically from the underlying lattice gauge theory at strong gauge couplings and heavy quark masses, and at leading order it has the form of an SU(3) spin model in $D=3$ dimensions ( $S_{P}$ has been computed to higher orders in the combined strong-coupling/hopping parameter expansion in [3]). This model has been solved at finite chemical potential $\mu$ by several different methods, including the flux representation [4], stochastic quantization [5], reweighting [3], and the mean field approach [6]. The motivation is that since the phase diagram for $S_{\text {spin }}$ has been determined over a large range of $J, h, \mu$ by the methods mentioned above, perhaps the same methods can be successfully applied to solve $S_{P}$, providing that theory is known in the parameter range (of temperature, quark mass, and chemical potential) of interest. The phase diagram of the effective theory will mirror the phase diagram of the underlying gauge theory.

Here we compute $S_{P}$ from an underlying $S U(3)$ lattice gauge theory at $\beta=5.4$ with staggered fermions of mass $m a=1.0$ on $L^{3} \times 4$ lattices with $L=16$, using the "relative weights" method introduced in $[7,8]$. We then deal with the sign problem via mean field theory, as shown in [6], which now seems to be a surprisingly accurate method for solving effective actions of this kind.

\section{The relative weights method}

The effective Polyakov line action $S_{P}$ of a lattice gauge theory is defined by integrating out all degrees of freedom of the lattice gauge theory, under the constraint that the Polyakov line holonomies are held fixed. It is convenient to implement this constraint in temporal gauge, where all timelike link variables are set equal to the identity except on some time slice, i.e. $U_{0}(\mathbf{x}, t \neq 0)=\mathbb{1}$. Then the timelike link variables at time slice $t=0$ are the Polyakov line holonomies, and we have

$$
\exp \left[S_{P}\left[U_{\vec{x}}\right]\right]=\int D U_{0}(\vec{x}, 0) D U_{k} D \phi\left\{\prod_{\vec{x}}\left[U_{\vec{x}}-U_{0}(\vec{x}, 0)\right]\right\} e^{S_{L}},
$$

where $\phi$ denotes any matter fields, scalar or fermionic, coupled to the gauge field, and $S_{L}$ is the lattice action (note that we adopt a sign convention for the Euclidean action such that the Boltzmann weight is proportional to $\exp [+S])$.

It is difficult to carry out the integration on the rhs of Eq.(2.1) directly. The relative weights approach is based on the observation that if $U_{\vec{x}}^{\prime}$ and $U_{\vec{x}}^{\prime \prime}$ are two Polyakov line configurations which are nearby in configuration space, then the ratio

$$
\exp \left[\Delta S_{P}\right]=\frac{\int D U_{k} D \phi e^{S_{L}^{\prime}}}{\int D U_{k} D \phi e^{S_{L}^{\prime \prime}}}=\frac{\int D U_{k} D \phi \exp \left[S_{L}^{\prime}-S_{L}^{\prime \prime}\right] e^{S_{L}^{\prime \prime}}}{\int D U_{k} D \phi e^{S_{L}^{\prime \prime}}}=\left\langle\exp \left[S_{L}^{\prime}-S_{L}^{\prime \prime}\right]\right\rangle
$$

is easily computed as an expectation value, by standard methods. Here $S_{L}^{\prime}, S_{L}^{\prime \prime}$ correspond to the lattice action with timelike link variables $U_{0}(\vec{x}, 0)$ at $t=0$ fixed to $U_{\vec{x}}^{\prime}, U_{\vec{x}}^{\prime \prime}$ respectively. Let $U_{\vec{x}}(\lambda)$ be a path through Polyakov line configuration space, parametrized by $\lambda$. Defining $U_{\vec{x}}^{\prime}=U_{\vec{x}}\left(\lambda_{0}+\right.$ $\Delta \lambda / 2)$ and $U_{\vec{x}}^{\prime \prime}=U_{\vec{x}}\left(\lambda_{0}-\Delta \lambda / 2\right)$ we can easily compute $\Delta S_{P}=S_{P}\left[U_{\vec{x}}^{\prime}\right]-S_{P}\left[U_{\vec{x}}^{\prime \prime}\right]$ and we are able 
to extract the directional derivative $d S_{P} / d \lambda$ at any point along the path. By a judicious choice of derivatives, it is possible to compute $S_{P}$ itself at zero (or at finite imaginary) chemical potential.

\section{The effective Polyakov line action}

For heavy quarks one can use the hopping parameter expansion to expand the log of the fermionic determinant in terms of loops. The terms which depend on chemical potential are those terms which wind around the lattice, and at each order in fugacity $\varepsilon^{\mu / T}$, to leading order in the hopping parameter $\kappa$, these are the traces of Wilson lines winding multiple times around the lattice in the time direction. If we consider only the single winding terms (which are simply Polyakov lines), we have the contribution

$$
h \sum_{\vec{x}}\left(e^{\mu / T} \operatorname{Tr} U_{\vec{x}}+e^{-\mu / T} \operatorname{Tr} U_{\vec{x}}^{\dagger}\right)
$$

where $U_{\vec{x}}$ is the Polyakov line holonomy $\left(P_{\vec{x}}=\frac{1}{3} \operatorname{Tr} U_{\vec{x}}\right)$, with $h=(2 \kappa)_{t}^{N}$, with $\kappa$ the hopping parameter for Wilson fermions, or $1 / 2 m$ for staggered fermions, and $N_{t}$ is the extension of the lattice in the time direction. In [3] it is shown that one can go a little further and sum up all the multiple winding terms which gives us, to leading order in $\kappa$, the piece of the fermionic contribution that depends on $\mu$ :

$$
\exp \left[S_{F}(\mu)\right]=\sum_{\vec{x}} \operatorname{det}\left[1+h e^{\mu / T} \operatorname{Tr} U_{\vec{x}}\right]^{p} \operatorname{det}\left[1+h e^{-\mu / T} \operatorname{Tr} U_{\vec{x}}^{\dagger}\right]^{p}
$$

where determinants can be expressed entirely in terms of Polyakov line operators, using the identities

$$
\begin{aligned}
\operatorname{det}\left[1+h e^{\mu / T} \operatorname{Tr} U_{\vec{x}}\right] & =1+h e^{\mu / T} \operatorname{Tr} U_{\vec{x}}+h^{2} e^{2 \mu / T} \operatorname{Tr} U_{\vec{x}}^{\dagger}+h^{3} e^{3 \mu / T}, \\
\operatorname{det}\left[1+h e^{-\mu / T} \operatorname{Tr} U_{\vec{x}}^{\dagger}\right] & =1+h e^{-\mu / T} \operatorname{Tr} U_{\vec{x}}^{\dagger}+h^{2} e^{-2 \mu / T} \operatorname{Tr} U_{\vec{x}}+h^{3} e^{-3 \mu / T} .
\end{aligned}
$$

This is the full fermionic contribution for heavy $(\kappa \ll 1)$ and dense $e^{\mu / T} \gg 1$ quarks. The power is $p=1$ for four flavors of staggered fermions, and $p=2 N_{f}$ for $N_{f}$ flavors of Wilson fermions. The parameter $h$ depends on the bare quark mass and the gauge coupling. We are going to make the strong assumption that this form of the fermionic determinant remains approximately valid up to the high- $T$ transition or crossover, for some choice of $h$, even in a regime where the hoppingparameter/strong-coupling expansion breaks down. Another approximation is that we are neglecting center symmetry-breaking products of Polyakov lines $P_{\vec{x}} P_{\vec{y}}$ (and complex conjugates) at $\vec{x} \neq \vec{y}$, which are generated by double-winding, non-overlapping Polyakov loops. Taken everything together, the full effective action motivated by the heavy-dense action, that we want to determine from relative weights, should look like this:

$$
\begin{aligned}
S_{\text {eff }}\left[U_{\vec{x}}\right] & =\sum_{\vec{x}, \vec{y}} P_{\vec{x}} K(x-y) P_{\vec{y}} \\
& +p \sum_{\vec{x}} \log \left(1+h e^{\mu / T} \operatorname{Tr}\left[U_{\vec{x}}\right]+h^{2} e^{2 \mu / T} \operatorname{Tr}\left[U_{\vec{x}}^{\dagger}\right]+h^{3} e^{3 \mu / T}\right) \\
& +\log \left(1+h e^{-\mu / T} \operatorname{Tr}\left[U_{\vec{x}}\right]+h^{2} e^{-2 \mu / T} \operatorname{Tr}\left[U_{\vec{x}}^{\dagger}\right]+h^{3} e^{-3 \mu / T}\right)
\end{aligned}
$$


We first run a standard Monte Carlo simulation, generate a configuration of Polyakov line holonomies $U_{\vec{x}}$, and compute the Polyakov lines $P_{\vec{x}}$. Then, we look at the Fourier (or "momentum") components $a_{\vec{k}}=a_{\vec{k}}^{R}+i a_{\vec{k}}^{I}$ of Polyakov line configurations $P_{\vec{x}}=\sum_{\vec{k}} a_{\vec{k}} e^{i \vec{k} \cdot \vec{x}}$, and compute the derivatives with respect to the real part of $a_{\vec{k}}$

$$
\frac{1}{L^{3}}\left(\frac{\partial S_{P}}{\partial a_{\vec{k}}^{R}}\right)_{a_{\vec{k}}^{R}=\alpha}
$$

as a function of the lattice momentum $k_{L}=2 \sqrt{\sum_{i=1}^{3} \sin ^{2}\left(k_{i} / 2\right)}$, with components $k_{i}=2 \pi m_{i} / L$, using the following triplets $\vec{m}=\left(m_{1} m_{2} m_{3}\right)$ of mode numbers:

$$
\begin{array}{r}
(000),(100),(110),(200),(210),(300),(311),(400),(322),(430), \\
(333),(433),(443),(444),(554) .
\end{array}
$$

We set the momentum mode $a_{\vec{k}}=0$ in this configuration to zero, to obtain the configuration

$$
\widetilde{P}_{\vec{x}}=P_{\vec{x}}-\left(\frac{1}{L^{3}} \sum_{\vec{y}} P_{\vec{y}} e^{-i \vec{k} \cdot \vec{y}}\right) e^{i \vec{k} \cdot \vec{x}} .
$$

Then we define

$$
P_{\vec{x}}^{\prime \prime}=\left(\alpha-\frac{1}{2} \Delta \alpha\right) e^{i \vec{k} \cdot \vec{x}}+f \widetilde{P}_{x} \quad \text { and } \quad P_{\vec{x}}^{\prime}=\left(\alpha+\frac{1}{2} \Delta \alpha\right) e^{i \vec{k} \cdot \vec{x}}+f \widetilde{P}_{x},
$$

where $f$ is a constant close to one; in practice we have used $f=0.8$, the choice $f=1$ is only possible in the large volume, $\alpha \rightarrow 0$ limit. From $P_{\vec{x}}^{\prime \prime}, P_{\vec{x}}^{\prime}$ we construct the holonomy configurations $U_{\vec{x}}^{\prime \prime}, U_{\vec{x}}^{\prime}$, and compute the observable (3.5) via the relative weights method specified in Eq. (2.2), keeping the holonomies fixed during Monte Carlo simulations. We average results over 2000 measurements on 20 different configurations for each $k$ value in (3.6) and different $\alpha=0.01-0.06$.

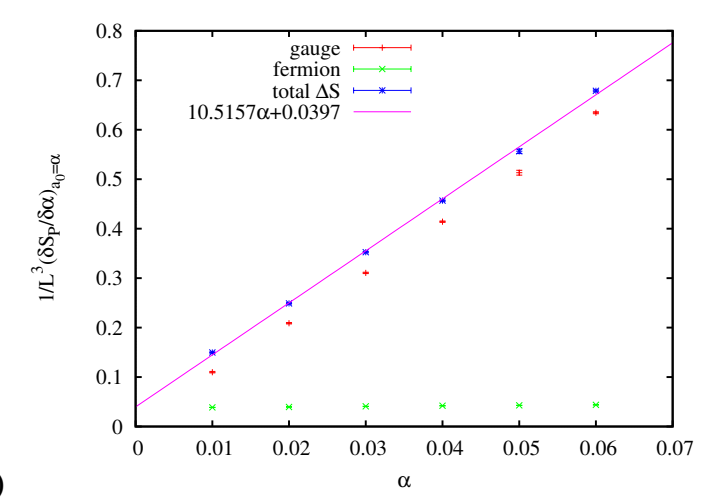

b)

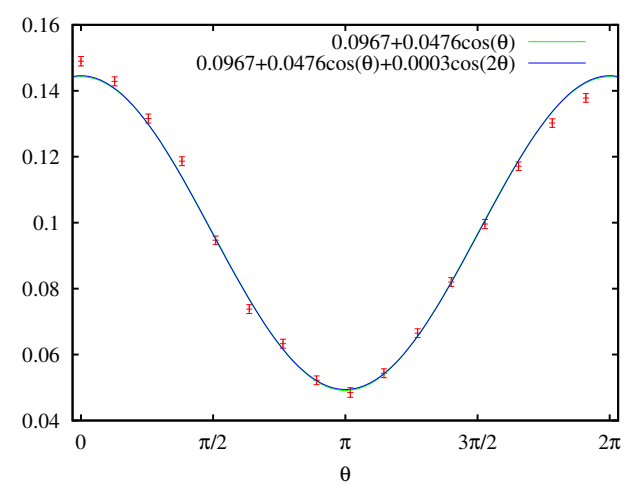

Figure 1: Derivatives of $S_{P}$ with respect to momentum modes $a_{\vec{k}}$, evaluated at $a_{\vec{k}}=\alpha$ for the zero mode $k=0$ : (a) data points fit by a straight line of slope $2 \widetilde{K}(0) \alpha$, (b) plotted against the imaginary chemical potential $\mu / T=i \theta$; note that the negligible $\cos (2 \theta)$ term supports the neglect of center symmetry-breaking terms, at $m a=1$, which are quadratic in the Polyakov lines. 
In order to extract the kernel $K(x-y)$ we start with fitting the kernel $\widetilde{K}(k)$ in momentum space and $h$ to the lattice data for the $k=0$ mode, as shown in Fig. 1a) via

$$
\frac{1}{L^{3}}\left(\frac{\partial S_{P}}{\partial a_{\vec{k}}}\right)_{a_{\vec{k}}=\alpha}=2 \widetilde{K}(k) \alpha+\frac{p}{L^{3}} \sum_{x}\left(3 h e^{i k x}+3 h^{2} e^{-i k x}+\text { c.c. }\right) .
$$

For a more precise extraction of $\widetilde{K}(0)$ and $h$ we also carry out the relative weights calculation in a lattice gauge theory with an imaginary chemical potential $\mu / T=i \theta$. This is done by simply multiplying the fixed configurations $U_{\vec{x}}^{\prime}, U_{\vec{x}}^{\prime \prime}$ of timelike links at $t=0$ by an $\vec{x}$-independent phase factor $e^{i \theta}$, and calculating the derivatives of $S_{P}$ wrt $\theta$ at a set of $\theta$ values and fixed $\alpha$ values (see Fig. 1(b) for $\alpha=0.01$ ). We fit this data for each $\alpha$ to

$$
2 \widetilde{K}(0) \alpha+\frac{p\left(3 h e^{i \theta}+3 h^{2} e^{2 i \theta}\right)}{L^{3} \sum_{x}\left(1+3 h e^{i \theta} P_{\vec{x}}+3 h^{2} e^{2 i \theta} P_{\vec{x}}^{\dagger}+h^{3} e^{3 i \theta}\right.}+c . c .
$$

and extrapolate the results for $h$ and $\widetilde{K}(0)$ to $\alpha \rightarrow 0$. Having computed $h$ we use Eq. (3.9) again to fit $\widetilde{K}(k)$ for $k \neq 0$ to the lattice data at zero chemical potential again. We find that the extracted data of the kernel $\widetilde{K}(k)$ almost fits two straight lines, defined by some $\widetilde{K}^{f i t}\left(k_{L}\right)$, except for $\widetilde{K}(0)$, as shown in Fig. 2a). Next, we calculate the position-space kernel with a long distance cutoff $r_{\max }$

$$
K(\vec{x}-\vec{y})=\left\{\begin{array}{cc}
\frac{1}{L^{3}} \sum_{\vec{k}} \widetilde{K}^{f i t}\left(k_{L}\right) e^{\vec{k} \cdot(\vec{x}-\vec{y})} & |\vec{x}-\vec{y}| \leq r_{\max } \\
0 & |\vec{x}-\vec{y}|>r_{\max }
\end{array} .\right.
$$

The cutoff $r_{\max }$ is chosen so that, upon transforming this kernel back to momentum space, the resulting $\widetilde{K}(k)$ also fits the low-momentum data at $k=0$, as shown in Fig. $2 \mathrm{~b}$ ).
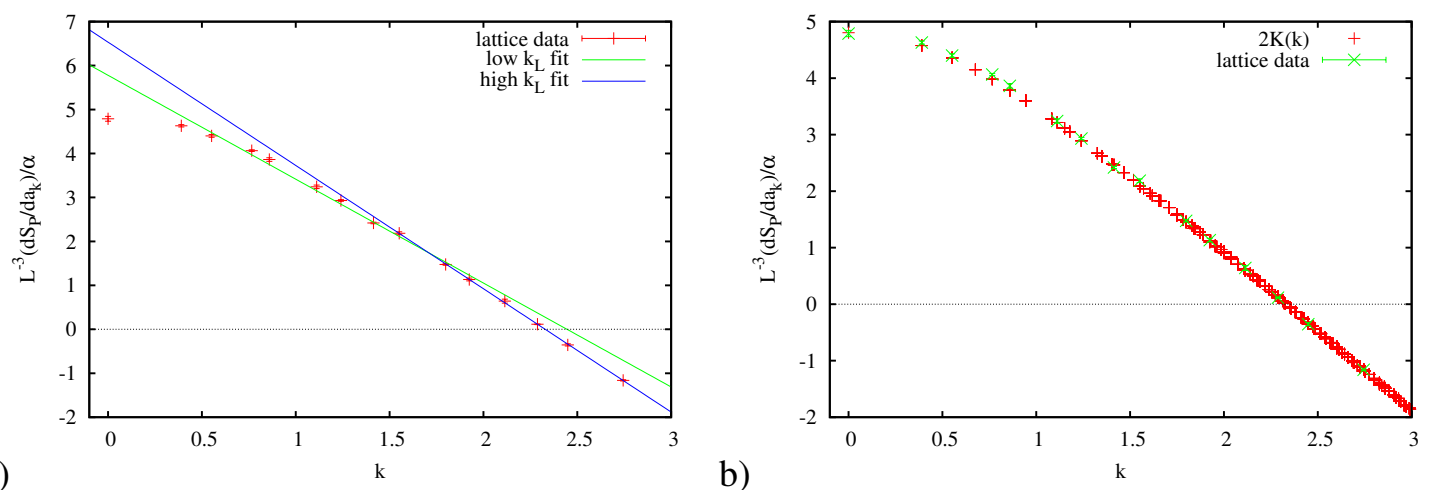

Figure 2: Derivatives of $S_{P}$ with respect to momentum modes $a_{\vec{k}}$, evaluated at $a_{\vec{k}}=\alpha$ for 15 values of $k_{L}$ : (a) data points fit by two straight lines. (b) the data points together with $2 \widetilde{K}\left(k_{L}\right)$, determined by the procedure explained in the text.

Now that we have obtained the kernel $K(\vec{x}-\vec{y})$ and $h$ we can simulate the effective PLA (3.4) at $\mu=0$ by standard lattice Monte Carlo methods. We calculate the correlator

$$
G(R)=\frac{1}{N^{2}}\left\langle\operatorname{Tr}\left[P_{\vec{x}}\right] \operatorname{Tr}\left[P_{\vec{y}}^{\dagger}\right]\right\rangle, R=|\vec{x}-\vec{y}|
$$



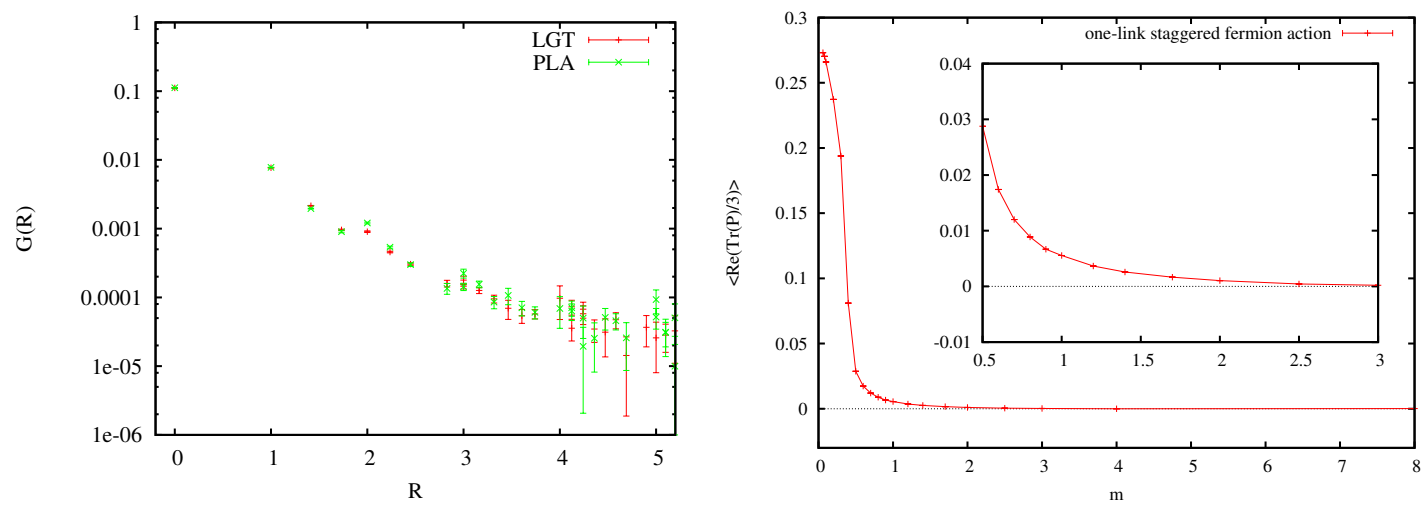

Figure 3: (a) The Polyakov line correlators computed from numerical simulation of the effective PLA $S_{P}$, and from simulation of the underlying lattice $\mathrm{SU}(3)$ gauge theory at $\beta=5.4$ with staggered fermions of mass $m a=1$ on $16^{3} \times 4$ lattices. (b) The VEV of the Polyakov lines in the gauge theory, vs. mass $m$ in lattice units.

and compare it with the corresponding Polyakov line correlator computed in the underlying SU(3) lattice gauge theory at $\beta=5.4$ with staggered fermions of mass $m a=1$ on $16^{3} \times 4$ lattices. The comparison (including off-axis separations) is shown in Fig. 3a). Allowing for the fact that the data is a little noisy beyond $R=4$, this seems like good agreement. Fig. 3b) shows the expectation value (VEV) of the Polyakov lines in the lattice gauge theory at $\beta=5.4$, showing a phase transition below $m a=1$. The PLA simulation reproduces the correct Polyakov correlators (and VEV) in the higher mass region above the phase transition, however, going to smaller quark masses we find discrepancies, indicating that our ansatz for $S_{P}$ is inadequate beyond the transition/crossover.

\section{Preliminary Results at Finite Density}

At finite $\mu$ there is still a sign problem in the effective action (3.4), and this problem we address via the mean field approach developed in [6]. The treatment of $S U(3)$ spin models at finite $\mu$ is a minor variation of standard mean field theory at zero chemical potential. For details of the approach, and comparison to complex Langevin see [6]. The results of the mean field calculation at $\beta=5.4$ and $N_{t}=4$ for staggered quarks of mass $m a=1$, using the extracted parameter $h$, are shown in Fig. 4. The VEVs of Polyakov lines $\langle\operatorname{Tr} U\rangle$ and $\left\langle\operatorname{Tr} U^{\dagger}\right\rangle$ in Fig. 4b) reproduce the correct value at $\mu=0$ and go to 0 for large $\mu / T$, while the number density in Fig. 4b) saturates at $n=3$ particles/lattice site, as required by the Pauli exclusion principle for staggered quarks with three color. There is no phase transition for $m a=1$, as in the heavy quark case.

\section{Conclusions \& Outlook}

We have presented the relative weights method for extracting the effective Polyakov line action from $\mathrm{SU}(3)$ lattice gauge theory at $\beta=5.4$ with dynamical staggered fermions of mass $m a=1$ on $16^{3} \times 4$ lattices. We find good agreement between Polyakov line correlators computed in the effective action and in the underlying gauge theory at zero chemical potential. Mean field methods 

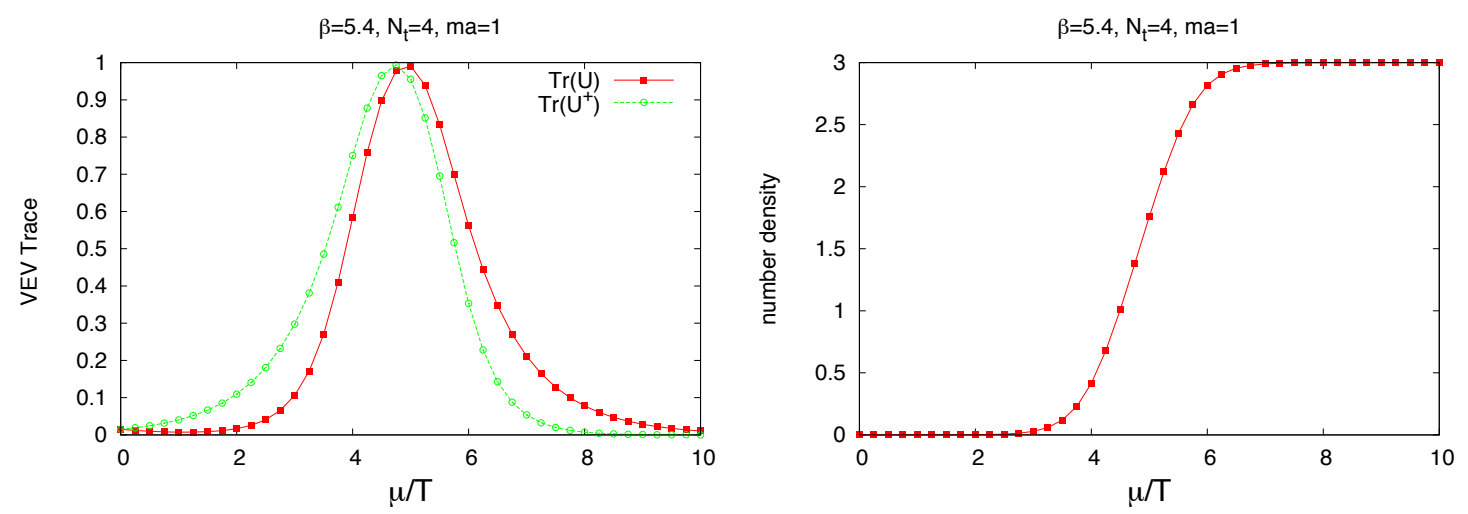

Figure 4: Mean field solution of the effective Polyakov line action $S_{P}$ corresponding to a gauge theory at $\beta=5.4$ with staggered quarks of mass $m a=1,16^{3} \times 4$, at finite values of the chemical potential. (a) the expectation value of Polyakov lines $\langle\operatorname{Tr} U\rangle$ and $\left\langle\operatorname{Tr} U^{\dagger}\right\rangle$ vs. $\mu$; (b) particle number density vs. $\mu$.

have been employed to determine the expectation value of observables in the effective action as a function of chemical potential.

The next step is to proceed to lighter quarks. In this case we may have to fit our data to a more complicated action. If the results at $\mu=0$ pass our usual tests, then we will attempt to locate phase transitions in the QCD phase diagram, which is the ultimate goal.

\section{References}

[1] P. de Forcrand, "Simulating QCD at finite density," PoS LAT2009 (2009) 010, arXiv: 1005.0539 [hep-lat].

[2] G. Aarts, "Complex Langevin dynamics and other approaches at finite chemical potential," $P o S$

LATTICE2012 (2012) 017, arXiv:1302.3028 [hep-lat] .

[3] M. Fromm, J. Langelage, S. Lottini, and O. Philipsen, "The QCD deconfinement transition for heavy quarks and all baryon chemical potentials," JHEP 01 (2012) 042, arXiv: 1111.4953 [hep-lat].

[4] Y. D. Mercado and C. Gattringer, "Monte Carlo simulation of the SU(3) spin model with chemical potential in a flux representation," Nucl. Phys. B862 (2012) 737-750, arXiv:1204.6074 [hep-lat].

[5] G. Aarts and F. A. James, "Complex Langevin dynamics in the SU(3) spin model at nonzero chemical potential revisited," JHEP 01 (2012) 118, arXiv:1112.4655 [hep-lat] .

[6] J. Greensite and K. Splittorff, "Mean field theory of effective spin models as a baryon fugacity expansion," Phys. Rev. D86 (2012) 074501, arXiv:1206.1159 [hep-lat] .

[7] J. Greensite and K. Langfeld, "Effective Polyakov line action from the relative weights method," Phys.Rev. D87 (2013) 094501, arXiv:1301.4977 [hep-lat] .

[8] J. Greensite and K. Langfeld, "Effective Polyakov line action from strong lattice couplings to the deconfinement transition," Phys.Rev. D88 (2013) 074503, arXiv:1305.0048 [hep-lat] . 